\title{
Subjective memory complaints in Italian elderly with mild cognitive impairment: implication of psychological status
}

\author{
Cinzia Giuli $^{1} \cdot$ Paolo Fabbietti $^{2} \cdot$ Cristina Paoloni $^{1} \cdot$ Mirko Pensieri $^{1}$ \\ Fabrizia Lattanzio $^{3}$ - Demetrio Postacchini ${ }^{1}$
}

Received: 16 February 2016/ Accepted: 15 March 2016/Published online: 30 March 2016

(c) The Author(s) 2016. This article is published with open access at Springerlink.com

\begin{abstract}
Subjective cognitive and memory complaints (SMC) are common in later life and are considered an indicator for progression to cognitive decline. The aim of the present study was to identify the relationship among SMC, neuropsychiatric symptoms and psychological aspects in elderly subjects with mild cognitive impairment (MCI) as well as to analyse the effect on SMC of a comprehensive cognitive training. Data from a sample of 94 patients enrolled in 'My Mind Project' (Grant No. 154/GR2009-1584108) were collected. The study evidenced that depression was a significant predictor of SMC and that after the training, the number of subjects with SMC was significantly reduced in the experimental group in comparison to the control one. These results suggest that the participation in cognitive stimulation protocols may improve the perception of SMC in subjects with MCI.
\end{abstract}

Keywords Subjective memory complaints · Elderly · Mild cognitive impairment - Cognitive training . Depression · Italy

Cinzia Giuli

c.giuli@inrca.it

1 Unit of Geriatrics, Italian National Research Center on Aging, INRCA, IRCCS, Contrada Mossa, 63900 Fermo, Italy

2 Biostatistical Centre, Italian National Research Center on Aging (INRCA), Ancona, Italy

3 Scientific Direction, Italian National Research Center on Aging, INRCA, Ancona, Italy

\section{Introduction}

The study of the role played by subjective memory complaints (SMC) in later life is a key topic. In particular, SMC have been considered a main criterion for evaluation and classification of mild cognitive impairment (MCI) [1] and are recognized by some authors as indicator for progression to dementia [2]. Contrarily, as reported in a recent study, some authors, suggested that SMC are not an essential criterion for MCI [3]. Yates et al. appointed that the possible reason for this discrepancy could include individual variation of elderly people in adapting to cognitive change where some individuals may not perceive such changes as significant or requiring actions. In particular, these authors evidenced that progression to dementia is better predicted by SMC than by global cognitive impairment or by domain-specific cognitive decline [3].

Elderly people are usually affected by perception of loss of cognitive functions than they were young [4]. In particular, SMC are common in the healthy older population, but often they may not be experienced by elderly with cognitive decline [1]. Some authors have stated that SMC may influence the relationship between mood and cognitive functioning [3] and may have a negative effect on wellbeing as complaints might reflect health and psychological disorders such as depression, perceived stress, and anxiety $[5,6]$.

Memory knowledge and objective memory performance can be effectively improved in old age through specific trainings, which include the use of mnemonic strategies [7, 8].

Given this background, the aims of this study were:

1. To analyse the predictors of SMC at baseline, taking into account the relationship among SMC, 
neuropsychiatric symptoms and psychological status in elderly patients with MCI;

2. To evaluate the immediate impact of a comprehensive intervention on SMC (phase of follow-up 1).

\section{Materials and methods}

\section{Subjects and recruitment}

Preliminary analyses were conducted in 94 Italian community-dwelling elderly subjects with multidomain MCI living in the Marche region, enrolled in the study 'My Mind Project: The effects of cognitive training for elderly' from the Evaluation of Alzheimer's Unit at the INRCA Hospital in Fermo. Eligibility was determined after a complete medical and neuropsychological examination to assess inclusion/exclusion criteria, as indicated in Casoli et al. [9]. The inclusion criteria included: age 65 years or older, availability during the training and testing phases, and presence of a caregiver. Exclusion criteria included: presence of serious medical or psychiatric conditions and sensori-motor deficits that would prevent participation in training, and presence of neurodegenerative disorders.

The study used a prospective randomized intervention protocol for the assessment of the effect of a comprehensive cognitive training. After the enrolment, subjects were randomly assigned to the experimental group (EG), whose members received the intervention (the detailed description is reported below) or to the control group (CG). Subjects with scores greater than or equal to 25 on the Memory Assessment Complaint Questionnaire (MAC-Q) of Crook were classified as 'Complainers' while the other subjects were defined as 'Non-complainers'. Four groups were identified: 'Complainers' and 'Non complainers' included into EG and CG.

All subjects were tested at baseline and at the end of intervention (follow-up) using a neuropsychological test battery including assessments of overall cognitive status, memory and learning processes, attention, language and executive functions, functional status, psycho-social aspects, lifestyle characteristics [10].

\section{Instruments}

\section{Assessment of SMC}

The MAC-Q was used to assess participants' memory complaints. It is a questionnaire for the assessment of daily activities and overall memory functioning comparing present moment to past.
Assessment of mood status

Researchers used the Geriatric Depression Scale (GDS-30) of Yesavage to measure participants' mood status.

\section{Assessment of perceived stress}

Participants' perceived stress levels were measured using the Perceived Stress Scale (PSS) of Cohen. It comprises 14 items and asks about feelings and thoughts during the last month.

\section{Assessment of functional status}

The Activities of Daily Living (ADL) of Katz and Instrumental Activities of Daily Living (IADL) of Lawton and Brody were used to assess participants' functional status.

\section{Intervention}

The EG received an individual comprehensive multi-modal intervention that included restorative and compensatory cognitive training, which consists of learning strategies for orientation, memory, categorisation and clustering. Because MCI subjects often present symptoms of anxiety, stress and/or depression due to their consciousness of cognitive decline, the potential influence of SMC was evaluated as they may affect the perception of cognitive decline. Some practical and compensation strategies were taught as well as aids and psychological support for stress and mood disease management to improve performance and psycho-education about memory loss. Advice and education on a healthy lifestyle was also provided. All participants were asked to perform cognitive and metacognitive at-home exercises each day. The intervention consisted of 10 sessions of about $45 \mathrm{~min}$, once a week.

\section{Statistical analysis}

Data were expressed as mean \pm standard deviation (continuous variables) or as percentage (categorical variables). Statistical comparisons were performed using the $t$-Student test or by Chi square test to compare Complainers and Noncomplainers in EG and CG at baseline and follow-up. To obtain an estimate of the independent association between study variables and outcomes, variables significantly distinguishing groups in preliminary analysis were entered into a logistic regression model; Pearson's coefficient was used to assess correlation among variables. The significance was accepted for $p<0.05$. All analyses were performed using SPSS V19.0 Statistical Software Package for Windows. 


\section{Results}

Comparisons of socio-demographic data and neuropsychological profile between EG and CG 'Complainers' and 'Non complainers' at baseline and follow up were reported in Tables $1,2$.

At baseline, $72.3 \%$ of MCI subjects had SMC. In particular, Complainers showed significant differences in comparison to Non-complainers in psychological and neuropsychiatric symptoms (mood and perceived stress), functional status (ADL, IADL) areas. In the whole sample at baseline, the GDS score was $6.46( \pm 3.63)$ and the PSS score was 16.23 ( \pm 6.80 ) for Non-complainers, whereas the scores for Complainers were significantly higher $(p<0.05)$ with a GDS score of $11.04( \pm 5.76)$ and a PSS score of 20.33 ( \pm 7.58$)$.

A multiple logistic regression analysis using the baseline data showed that the predictor of SMC in elderly with MCI was the depression status. Using the Hosmer-Lemeshow goodness of fit test, the results showed $\chi^{2}=10.342$, $p=0.242$, adjusting for gender, age, and score of the BADL, IADL, and PSS (Table 3).

After intervention, we observed a reduction of subjects with SMC in EG while no differences were found in CG (Tables 1, 2).

\section{Discussion}

Our preliminary results evidenced that subjects with depression have higher risk to have SMC, which is recognized as indicator for eventual progression to dementia $[2,6]$. The relationship between SMC and depression in
MCI is controversial and not fully clarified [11], thus also in our study further analysis are necessary. Indeed, both mood and cognitive decline could have a mutual relationship with SMC [1]. Some authors evidenced that SMC are more related to depression than objective cognitive decline and recently they appointed that SMC might play an important role on the relationship between MCI and mood [3]. These authors concluded that SMC in MCI could be a function of neuropsychiatric symptoms (such as depression) rather than being related to objective cognitive functions. In this our preliminary study we found that depression is a significant predictor of SMC. We choose to finalize intervention for MCI subjects also to psychological support for stress and mood disease management, as well as training to improve cognitive performances by means of the use of memory strategies which can compensate perceived memory failures [8]. Therefore, a metacognitive and motivational approach which improve self-esteem for elderly with MCI is necessary during cognitive training, as also indicated by other authors [4]. This study evidenced that the enhancement of cognitive functions is not sufficient to improve confidence of the elderly about their performance, because often they have many difficulties in modifying motivational attitudes. For this reason, often the beneficial effect of intervention may be limited in duration.

An interesting result is that EG had positive effects in terms of decrease of SMC. Our findings showed that the multidimensional and comprehensive intervention, as described above, could be effective for elderly people, in particular in terms of improvement in self perception of memory loss and SMC. We also observed improvement on some memory and other cognitive performances in EG as

Table 1 Characteristics of the experimental (EG) and the control (CG) group at baseline

\begin{tabular}{|c|c|c|c|c|c|c|}
\hline & \multicolumn{6}{|l|}{ Baseline } \\
\hline & \multicolumn{3}{|l|}{ EG } & \multicolumn{3}{|l|}{ CG } \\
\hline & $\begin{array}{l}\text { Non complainers } \\
N=8\end{array}$ & $\begin{array}{l}\text { Complainers } \\
N=38\end{array}$ & $p$ & $\begin{array}{l}\text { Non complainers } \\
N=18\end{array}$ & $\begin{array}{l}\text { Complainers } \\
N=30\end{array}$ & $p$ \\
\hline Age (years) & $75.88 \pm 5.24$ & $75.95 \pm 6.53$ & 0.974 & $76.67 \pm 6.32$ & $76.40 \pm 5.58$ & 0.267 \\
\hline Gender (female) & $5(62.5 \%)$ & $25(65.8 \%)$ & 0.859 & $11(61.1 \%)$ & $19(63.3 \%)$ & 0.878 \\
\hline \multicolumn{7}{|l|}{ Marital status } \\
\hline Married/cohabiting & $7(87.5 \%)$ & $22(57.9 \%)$ & 0.115 & $14(77.8 \%)$ & $22(73.3 \%)$ & 0.728 \\
\hline Widowed & $1(12.5 \%)$ & $16(42.1 \%)$ & & $4(22.2 \%)$ & $7(23.3 \%)$ & \\
\hline Other & $0(0.0 \%)$ & $0(0.0 \%)$ & & $0(0.0 \%)$ & $1(3.3 \%)$ & \\
\hline Education (years) & $5.75 \pm 1.38$ & $6.97 \pm 4.22$ & 0.426 & $5.88 \pm 3.96$ & $4.90 \pm 2.21$ & 0.273 \\
\hline GDS & $6.75 \pm 3.69$ & $11.18 \pm 6.41$ & $<0.05$ & $6.33 \pm 3.71$ & $10.86 \pm 4.92$ & 0.001 \\
\hline PSS & $18.25 \pm 7.90$ & $20.00 \pm 7.76$ & 0.581 & $15.33 \pm 6.29$ & $20.76 \pm 7.46$ & 0.01 \\
\hline ADL & $5.87 \pm 0.35$ & $5.81 \pm 0.39$ & 0.681 & $6.00 \pm 0.00$ & $5.73 \pm 0.44$ & $<0.05$ \\
\hline IADL & $8.00 \pm 0.00$ & $7.31 \pm 0.93$ & $<0.05$ & $7.55 \pm 0.70$ & $7.46 \pm 0.89$ & 0.705 \\
\hline
\end{tabular}


Table 2 Characteristics of the experimental (EG) and the control (CG) group at follow-up

\begin{tabular}{|c|c|c|c|c|c|c|}
\hline & \multicolumn{6}{|l|}{ Follow-up } \\
\hline & \multicolumn{3}{|l|}{ EG } & \multicolumn{3}{|l|}{ CG } \\
\hline & $\begin{array}{l}\text { Non complainers } \\
N=25\end{array}$ & $\begin{array}{l}\text { Complainers } \\
N=21\end{array}$ & $p$ & $\begin{array}{l}\text { Non complainers } \\
N=12\end{array}$ & $\begin{array}{l}\text { Complainers } \\
N=36\end{array}$ & $p$ \\
\hline Age (years) & $76.08 \pm 7.41$ & $76.29 \pm 4.84$ & 0.914 & $79.00 \pm 4.72$ & $75.89 \pm 5.91$ & 0.077 \\
\hline Gender (Female) & $17(68.0 \%)$ & $13(61.9 \%)$ & 0.665 & $6(50.0 \%)$ & $24(66.7 \%)$ & 0.302 \\
\hline \multicolumn{7}{|l|}{ Marital status } \\
\hline Married/cohabiting & $17(58.6 \%)$ & $12(47.1 \%)$ & 0.447 & $10(83.3 \%)$ & $26(72.2 \%)$ & 0.686 \\
\hline Widowed & $8(41.4 \%)$ & $9(52.9 \%)$ & & $2(16.7 \%)$ & $9(25.0 \%)$ & \\
\hline Other & $0(0.0 \%)$ & $0(0.0 \%)$ & & $0(0.0 \%)$ & $1(2.8 \%)$ & \\
\hline Education (years) & $6.16 \pm 3.37$ & $7.47 \pm 4.42$ & 0.271 & $5.83 \pm 3.95$ & $5.08 \pm 2.64$ & 0.549 \\
\hline GDS & $8.52 \pm 5.71$ & $11.28 \pm 6.13$ & 0.124 & $7.41 \pm 5.82$ & $11.36 \pm 4.54$ & $<0.05$ \\
\hline PSS & $16.60 \pm 8.42$ & $20.57 \pm 7.43$ & 0.097 & $16.58 \pm 9.29$ & $21.08 \pm 8.46$ & 0.156 \\
\hline $\mathrm{ADL}$ & $6.00 \pm 0.00$ & $5.61 \pm 0.49$ & $<0.001$ & $6.00 \pm 0.00$ & $5.77 \pm 0.42$ & 0.222 \\
\hline IADL & $7.44 \pm 1.00$ & $7.42 \pm 0.74$ & 0.965 & $7.58 \pm 0.79$ & $7.47 \pm 0.84$ & 0.111 \\
\hline
\end{tabular}

Table 3 Logistic regression analysis at baseline

\begin{tabular}{llllll}
\hline Dependent variables & OR & E.S. & $p$ & \multicolumn{2}{l}{$95 \%$ CI } \\
\cline { 5 - 6 } & & & & Lower & Upper \\
\hline Age & 0.961 & 0.049 & 0.415 & 0.873 & 1.058 \\
Gender & 1.951 & 0.580 & 0.249 & 0.626 & 6.080 \\
PSS & 1.017 & 0.043 & 0.693 & 0.934 & 1.107 \\
GDS & 1.194 & 0.078 & $<0.05$ & 1.025 & 1.391 \\
ADL & 0.337 & 1.122 & 0.332 & 0.037 & 3.038 \\
IADL & 0.664 & 0.416 & 0.325 & 0.294 & 1.502 \\
\hline
\end{tabular}

indicated in Balietti et al. [10], but this relationship will be deeply studied. Other authors [7] found that this kind of intervention can enhance the overall cognitive functioning and encoding as well as recalling of new verbal information of older adults with SMC with a durable effect in memory [12].

In conclusion, taking into account that subjects with depression and SMC are at high risk of dementia, the identification of these groups is important to analyze factors and interventions that could possibly contribute to the prevention of cognitive decline and symptoms related to cognitive deficits (such as depressive mood), as also indicated by other authors [13].

Further investigation is required to define more reliable factors, including biomarkers, to discriminate the relationship between depression and SMC and to identify the long-term effects of intervention on psychological status and neuropsychiatric symptoms.

Acknowledgments This work was supported by the "Ricerca Finalizzata" funded by the Italian Ministry of Health and the Marche Region (Grant Number 154/GR-2009-1584108).

\section{Compliance with ethical standards}

Conflict of interest No competing financial interests exist.

Ethical approval "All procedures performed in studies involving human participants were in accordance with the ethical standards of the institutional and/or national research committee and with the 1964 Helsinki declaration and its later amendments or comparable ethical standards." The study was approved by the Institutional Ethical Committee (code SC/12/301) of INRCA. Informed consent was obtained from all individual participants included in the study.

Open Access This article is distributed under the terms of the Creative Commons Attribution 4.0 International License (http://crea tivecommons.org/licenses/by/4.0/), which permits unrestricted use, distribution, and reproduction in any medium, provided you give appropriate credit to the original author(s) and the source, provide a link to the Creative Commons license, and indicate if changes were made.

\section{References}

1. Yates JA, Clare L, Woods RT, Matthews FE, Cognitive Function and Ageing Study Wales (2015) Subjective memory complaints are involved in the relationship between mood and mild cognitive impairment. J Alzheimers Dis 48:S115-S123. doi:10.3233/JAD150371

2. Rabin LA, Smart CM, Crane PK et al (2015) Subjective cognitive decline in older adults: an overview of self-report measures used across 19 international research studies. J Alzheimers Dis 1:S63S86. doi:10.3233/JAD-150154

3. Yates JA, Clare L, Woods RT, MRC CFAS (2015) Subjective memory complaints, mood and MCI: a follow-up study. Aging Ment Health 2:1-9. doi:10.1080/13607863.2015.1081150

4. Carretti B, Borella E, Zavagnin M, De Beni R (2011) Impact of metacognition and motivation on the efficacy of strategic memory training in older adults: analysis of specific, transfer and maintenance effects. Arch Gerontol Geriatr 52:e192-e197. doi:10.1016/j.archger.2010.11.004 
5. Zuniga KE, Mackenzie MJ, Kramer A, McAuley E (2016) Subjective memory impairment and well-being in communitydwelling older adults. Psychogeriatrics 16:20-26. doi:10.1111/ psyg. 12112

6. Lee PL (2014) The relationship between memory complaints, activity and perceived health status. Scand J Psychol 55:136-141. doi:10.1111/sjop.12107

7. Miller KJ, Siddarth P, Gaines JM et al (2012) The memory fitness program: cognitive effects of a healthy aging intervention. Am J Geriatr Psychiatry 20:514-523. doi:10.1097/JGP.0b013e318227 f821

8. Aronov A, Rabin LA, Fogel J et al (2015) Relationship of cognitive strategy use to prospective memory performance in a diverse sample of nondemented older adults with varying degrees of cognitive complaints and impairment. Neuropsychol Dev Cogn B Aging Neuropsychol Cogn 22:486-501. doi:10.1080/ 13825585.2014.984653

9. Casoli T, Giuli C, Balietti M, Giorgetti B, Solazzi M, Fattoretti P (2014) Effect of cognitive training on the expression of brain- derived neurotrophic factor in lymphocytes of mild cognitive impairment patients. Rejuvenation Res 17:235-238. doi:10.1089/ rej.2013.1516

10. Balietti M, Giuli C, Fattoretti P, Fabbietti P, Postacchini D, Conti F (2016) Cognitive stimulation modulates platelet total phospholipases A2 activity in subjects with mild cognitive impairment. J Alzheimers Dis 50:957-962. doi:10.3233/JAD-150714

11. Balash Y, Mordechovich M, Shabtai H, Giladi N, Gurevich T, Korczyn AD (2013) Subjective memory complaints in elders: depression, anxiety, or cognitive decline? Acta Neurol Scand 127:344-350. doi:10.1111/ane.12038

12. Kwok T, Wong A, Chan G et al (2013) Effectiveness of cognitive training for Chinese elderly in Hong Kong. Clin Interv Aging 8:213-219. doi:10.2147/CIA.S38070

13. Kryscio RJ, Abner EL, Cooper GE et al (2014) Self-reported memory complaints: implications from a longitudinal cohort with autopsies. Neurology 83:1359-1365. doi:10.1212/WNL.0000000000000856 